\title{
Lack of control enhances accurate and inaccurate identification responses to degraded visual objects
}

\author{
Manila Vannucci • Giuliana Mazzoni • Giulia Cartocci
}

Published online: 17 March 2011

(C) Psychonomic Society, Inc. 2011

\begin{abstract}
Recent studies have shown that lack of control induces illusory pattern perception. In this study, we demonstrate that lacking control also affected identification responses to degraded pictures of visual objects. As compared with control participants, participants in whom lack of control was experimentally induced produced identification responses to visual objects at a significantly higher level of degradation of the stimulus. Lacking control did not influence accuracy of identification, since the effect was present for both correct and incorrect identification responses, and did not encourage pure random guessing. Our results indicate that lacking control makes individuals more prone to achieve visual closure by attempting earlier identifications of perceptually degraded visual objects. Possible mechanisms involved in this effect are discussed.
\end{abstract}

\section{Keywords Object recognition - Visual perception}

Humans have a remarkable ability to categorize and identify visual objects and to recognize familiar objects even when only partial or distorted information is available. Extensive behavioral, electrophysiological, and neuroimaging studies in healthy subjects and neuropsychological

M. Vannucci $(\bowtie) \cdot G$. Cartocci

Department of Psychology, University of Florence,

Via San Salvi 12, Padiglione 26,

Florence, Italy

e-mail: manila.vannucci@psico.unifi.it

M. Vannucci

e-mail: mvannucci@tin.it

G. Mazzoni

Department of Psychology, University of Hull,

Hull, UK patients have shown that perceptual and cognitive factors related to the stimulus, task, and perceiver affect visual object recognition. The multilevel system that mediates object identification is flexible and malleable, and accurate object identification occurs when the best match between the perceptual representation of the visual object and the long-term memory representations of its visual structure is selected (Schendan \& Kutas, 2002; Ullman, 1996).

The mechanisms involved in object-model selection and the properties of the long-term stored object representations are modulated not only by bottom-up variables, such as perceptual characteristics of the visual objects (e.g., orientation, Jolicœur, 1985; Tarr \& Pinker, 1989; Vannucci \& Viggiano, 2000; color, Oliva \& Schyns, 2000; Tanaka \& Presnell, 1999), its semantic features (for a review, see Martin, 2007), or specific task constraints (Dickerson \& Humphreys, 1999; Hamm \& McMullen, 1998), but also by top-down processes. For example, identification of visual objects, especially when the visual stimulus is impoverished and degraded, is affected by the person's expertise (Viggiano, Righi, \& Galli, 2006), differences in visual object imagery abilities (Vannucci, Mazzoni, Chiorri, \& Cioli, 2008), and motivations, needs, and expectations (Bruner \& Goodman, 1947; Bruner \& Postman, 1947; Levine, Chein, \& Murphy, 1942). In this study, we investigated whether and how the process of identification of visual objects is affected by the person's need for control and whether this influence results in a better use of the physical information provided in the visual input.

Recent evidence has shown that experimentally created need for control induces illusory perception (i.e., completely inaccurate detection) of visual patterns in unstructured white noise (Whitson \& Galinsky, 2008, Experiment 3) and of a modified version of the snowy picture task (Whitson \& Galinsky, 2008, Experiment 2). Need for control was 
experimentally induced in those studies as a transitory condition by manipulating lack of control. This manipulation led to an increase in illusory pattern perception, making participants more likely to perceive a structure where no pattern was present. The authors explained this tendency as a compensatory mechanism induced by the distressing experience of lacking control. The need to be and to feel in control is so strong that individuals try to gain a sense of control at a perceptual/cognitive level, as a way of making sense of events and making the events predictable by providing them with an illusory structure. In line with this explanation, in the same series of experiments, the authors found that a reduction of this distressing state obtained via a standard self-affirmation procedure (i.e., letting individuals contemplate and affirm their important values) reduced the tendency to see illusory patterns. Thus, illusory perceptions seem to occur in response to the need to compensate for objective lack of control.

We hypothesized that experimentally induced lack of control also affects the processing of real visual objects. The data reported by Whitson and Galinsky (2008) do not provide an answer to this question. When actual objects were presented in their study, they were presented without time constraints on their identification. For this reason, the objects presented were always correctly identified by participants, leaving no room for any effect of lack of control. In the present study, we employed a visual object identification task with degraded pictures of real objects (Vannucci, Viggiano, \& Argenti, 2001). These are structured and meaningful pictures, but they are presented starting from the highest level of nine decreasing levels of degradation (see Fig. 1), obtained by manipulating the spatial frequency content of the visual stimulus, which is the primary physical factor analyzed by the human visual system for identifying real objects. Different ranges of spatial frequencies provide different kinds of information on the visual stimulus. The luminance blobs carried in low spatial frequencies (LFs) do not provide local details or texture features, but only a general skeleton of the image, whereas high spatial frequencies (HFs) convey information on metric part differences and texture patterns, in addition to making it possible to extract the general shape of the object (Collin, 2006; Hughes, Nozawa, \& Kitterle, 1996). By presenting stimuli with increasing levels of HFs (following a coarse-to-fine order), it is possible to calculate the mean level of filtering at which identification responses are provided and to distinguish between correct and incorrect identifications (Vannucci et al., 2001).

In previous studies, it was found that degraded pictures shown in an ascending order elicit both correct and incorrect identifications. The latter consisted in perceptually similar visual objects (e.g., drill and hair dryer; Bruner \& Potter, 1964; Lindfield \& Wingfield, 1999; Lindfield, Wingfield, \& Bowles, 1994; Snodgrass \& Hirshman, 1991).

Given the reduced role of bottom-up processes in the identification of degraded stimuli, this is the perfect task for investigating whether need for control, a top-down process, affects identification responses. On the basis of the results of Experiments 2 and 3 by Whitson and Galinsky (2008), we expected that experiencing experimentally induced need for control would lead participants to recognize a meaningful structure in the visual stimulus more easily and earlier - that is, at lower levels of filtering. More specifically, our paradigm makes it possible to test two different effects of lack of control on identification attempts.

First, lack of control might facilitate early visual closure and enhance the detection of a meaningful structure in

Fig. 1 Examples of an ascending sequence of nine filtering levels of a stimulus (a drill)

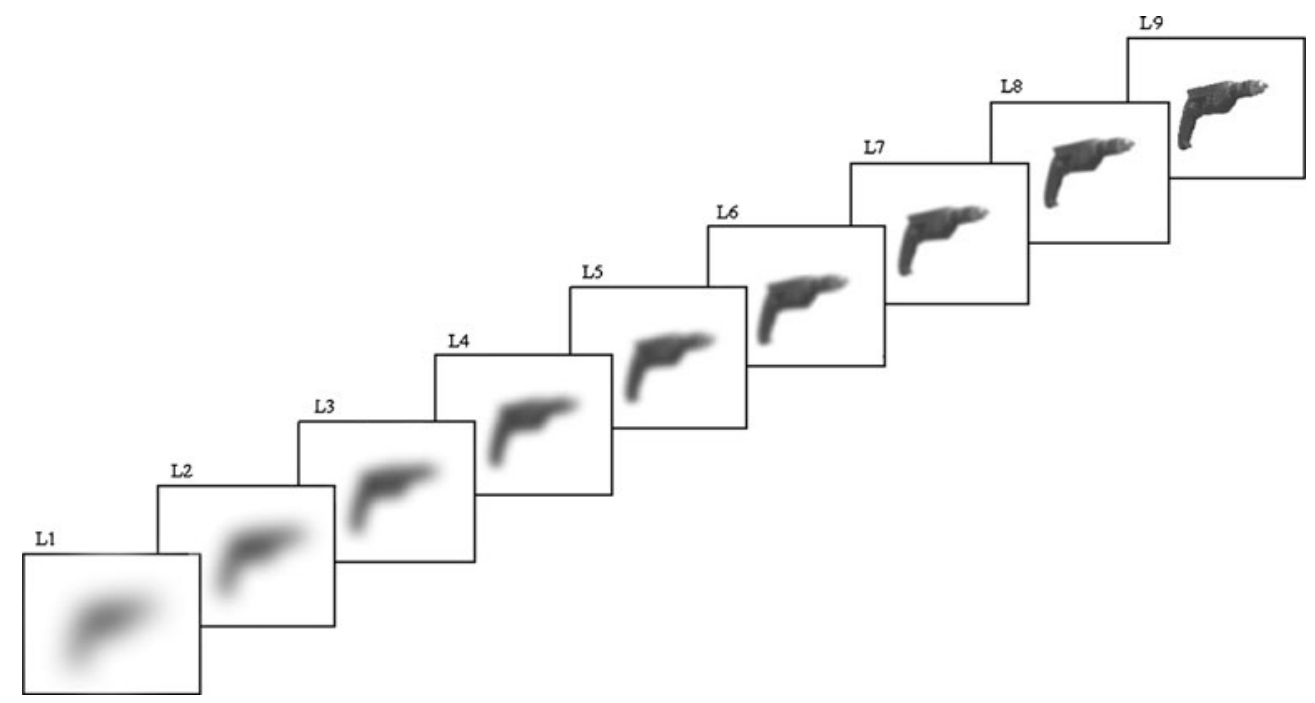


degraded pictures, resulting in more identification attempts (including both correct and incorrect identification responses) at higher levels of degradation.

Second, lack of control might selectively facilitate correct identification attempts, leading to more correct responses at early (more degraded) levels.

The present paradigm also makes it possible to examine whether lack of control might, instead, simply enhance random guessing, because of the necessity to provide an identification response without taking into account the content of the physical visual information. In this case, one would expect more incorrect responses in the lack-of-control group than in the control group for highly degraded pictures (very early levels of filtering for which a correct identification is unlikely) and, thus, more overall false identification attempts in this condition than in the control condition.

Individuals might also differ in the extent to which they are motivated to structure their world. Individuals with high levels of desire for simple structure are globally motivated to establish a structure in their everyday life; they enjoy routines, prefer familiar social situations, and are troubled by ambiguous and unpredictable situations (Neuberg \& Newsom, 1993; Thompson, Naccarato, \& Parker, 1989). This might influence the manner in which they react to an experimentally induced state of lack of control. For this reason, we decided to control for individual differences in the desire for structure by matching participants on this dimension when randomizing them to experimental and control conditions.

\section{Method}

\section{Participants}

Need for structure was assessed in a mass testing session in which 134 introductory psychology students at the University of Florence completed the Personal Need for Structure (PNS) scale (Neuberg \& Newsom, 1993; Thompson et al., 1989), along with questionnaires unrelated to this study. Those with scores within 1 standard deviation of the sample mean $(M=38.2, S D=9.5)$ were subsequently invited to take part in an experiment on visual perception. Potential participants were not informed that they had been selected according to their PNS score. Forty-eight right-handed students ${ }^{1}$ with normal or corrected-to-normal vision (35 females; age: 18-24 years) agreed to take part in the experiment. They were matched on the basis of their PNS score and were randomly assigned to either the lack-of-

\footnotetext{
${ }^{1}$ Left-handed participants were not intentionally excluded from the sample, and their lack of inclusion was due to chance.
}

control group or the control group (mean PNS scores were $37.45, S D=5.96$, in the lack-of-control group and 39.08, $S D=6.57$, in the control group).

\section{Stimuli and procedure}

Personal need for structure scale This is the most frequently used instrument to measure individual differences in the desire for structure (Neuberg \& Newsom, 1993; Thompson et al., 1989). Participants are requested to respond on a 6-point scale, ranging from strongly disagree $(=1)$ to strongly agree $(=6)$, to 11 items. Higher scores reflect higher desire for structure.

Lack-of-control manipulation To manipulate lack of control, we employed the concept identification task used by Whitson and Galinsky (2008), initially proposed by Pittman and Pittman (1979). Participants in the lack-of-control condition were told to find the concept in common to a series of pairs of symbols presented on the computer screen. They were informed that computer feedback would have helped them find the correct concept by informing them as to whether their choice was correct or incorrect. After a practice trial with ten pairs of figures, participants completed four trials, each with ten pairs of figures. The computer feedback was random and not contingent on the person's responses (the feedback "correct" and "incorrect" each appeared $50 \%$ of the time). In fact, there was no concept to identify, and thus participants were unable to correctly guess an answer.

Participants in the control group were told that in order to provide a "base rate" of responses, they were to answer without receiving computer feedback and that they should provide their best guess as to what concept the computer had selected. They were told that their performance did not matter and that we simply wanted their instinctive responses.

Identification of spatially filtered objects Spatially filtered versions of 60 gray-level photographs of real-life objects belonging to different semantic categories (animals, tools, etc.) were presented (Viggiano, Vannucci, \& Righi, 2004). Each object was subjected to nine different levels of spatial filtering. Stimuli were blurred by removing various ranges of spatial frequencies from the original spectrum of the image. The multiresolution filter selected was the Gaussian mask that performs low-pass filtering (Vannucci et al., 2001). Each object was presented using the ascending method of limits, in a sequence of nine levels of filtering (see Fig. 1), starting from the most blurred (level 1) and gradually adding new ranges of HFs, until the original complete version is created (level 9). The order of presentation of the nine levels was always the same across 
objects and individuals. Each object was displayed at all nine resolution levels, regardless of the level at which identification occurred (an equal number of pictures were presented to each participant at each level of filtering).

The stimuli were presented in central vision, on a PC screen, subtending a visual angle of $7.5^{\circ}$ high by $7.5^{\circ}$ wide, for $200 \mathrm{~ms}$, with an interstimulus interval ranging from 1 to 2 s. Presentation order was counterbalanced across participants. Participants were asked to identify and name the object and were given feedback on the correctness of the name. They pressed one key when able to identify, another key when unable to do so. They were informed that superordinate names (e.g., "animal" for horse) were not acceptable. Errors followed by immediate self-correction were considered correct responses. Correctness of responses was assessed against dominant and nondominant names obtained in a previous study (Viggiano et al., 2004). Responses that differed from the dominant and nondominant names were considered false identification attempts (e.g., hair dryer to the picture of a drill). The actual task was preceded by a practice phase with stimuli that did not appear in the actual experiment.

\section{Results}

Overall, the first identification was correct for 40.5 (out of $60)$ objects, indicating that in the majority of the cases, participants were able to correctly identify visual objects the first time they provided a response. In the case of false identification attempts, almost all responses referred to visual objects that were perceptually similar to the ones presented (e.g., hair dryer or gun for a drill; ship for a shark; wolf for a fox). Perceptual similarity was measured using the pixel-to-pixel spatial correspondence between presented objects and standard pictures (same size) of the false identification attempts (measure of the Euclidean overlap developed by Laws \& Gale, 2002; see also Laws, Gale, \& Leeson, 2003). Most false identification attempts for a given object were the same across participants (e.g., in $94 \%$ of the cases, the false identification attempt for the screwdriver was toothbrush).

To assess the effect of lack of control on identification responses obtained at different levels of degradation, we compared the level of filtering at which the first identification occurred in the two groups, distinguishing between correct and false identification attempts. A 2 (identification attempts, correct vs. false) $\times 2$ (group) mixed model ANOVA conducted on the level of filtering at which the first identification attempts occurred revealed that false identification attempts occurred at lower levels of filtering $(M=2.93)$ than did correct identification attempts $(M=3.62), F(1,46)=69.44, p<.0001$, partial $\eta^{2}=.60$.
More important, participants in the lack-of-control condition made identification responses significantly earlier, at lower levels of filtering $(M=3.06)$, than did participants in the control group $(M=3.48), F(1,46)=4.07, p=.05$ partial $\eta^{2}=.08$. The interaction was not significant, $F<1$, indicating that lack of control induced earlier identification responses, but the effect was not significantly different for correct and false identification attempts.

The fact that the group in need of control detected a meaningful structure in the visual array earlier than the control group was confirmed by a complementary analysis. For the analysis, we calculated the percentage of first correct identifications (on the total of correct identifications) and the percentage of first incorrect identification attempts (on the total of incorrect identification attempts) that occurred at each of the nine levels of filtering. We then conducted a 9 (filtering level) $\times 2$ (identification attempts, correct vs. false) $\times 2$ (group) mixed model ANOVA using Greenhouse-Geisser correction. The main effect of filtering level was statistically significant, $F(8,368)=56.13$, $p<.0001$, partial $\eta^{2}=.50$, with more identification attempts occurring at level $3(23.36 \%)$ than at any other level. This main effect was qualified by a significant filtering level $\times$ group interaction, $F(8,368)=3.16, p=.019$, partial $\eta^{2}=.07$ (see Fig. 2). Post hoc tests (alpha of .01) showed that the difference between the two groups was significant at level $3(p<.005)$, where participants in the lack-ofcontrol condition made more identification attempts than did those in the control group, and at levels $4(p<.01)$ and $5(p<.05)$, where they made fewer identification attempts than did the control group. No significant effect was found at the remaining levels. The lack of differences in identification attempts between groups at later levels of filtering ( 6 and higher) is due to the fact that the majority of identification attempts (93\%) occur before that level.

The interaction between filtering level and identification attempts was also significant, $F(1,46)=14.55, p<.0001$, partial $\eta^{2}=.24$. As was expected, post hoc tests showed that more false than correct identification attempts occurred

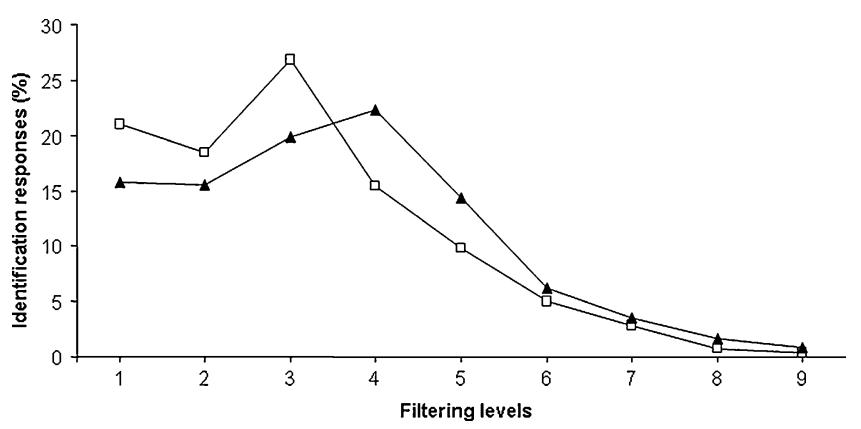

Fig. 2 First identification attempts (expressed as percentages) in the lack-of-control group (white squares) and in the control group (black triangle) as a function of filtering level 


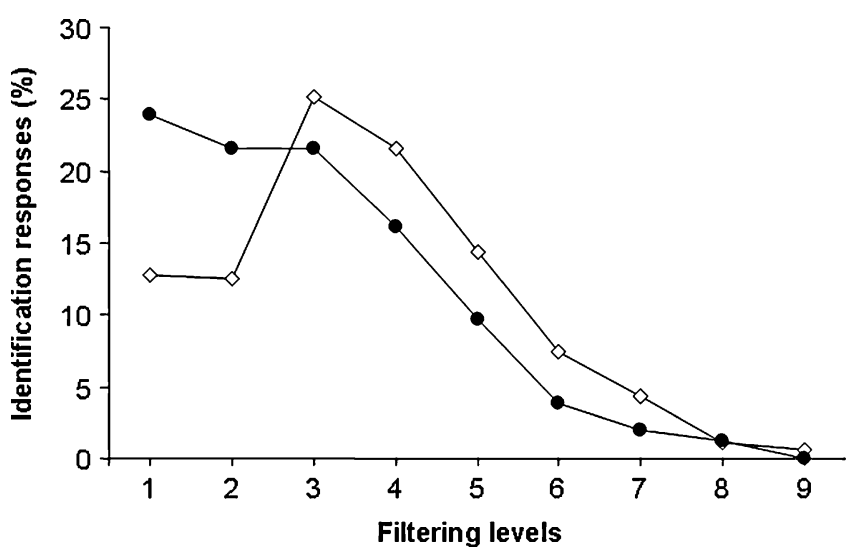

Fig. 3 Percentage of first correct (white squares) and incorrect (black dots) identification attempts as a function of filtering level

at levels $1(p<.0001)$ and $2(p<.0001)$, whereas at levels $4(p<.01), 5(p<.01), 6(p<.01)$, and $7(p<.01)$, there were more correct than false identification attempts (see Fig. 3).

Neither the two-way group $\times$ identification attempts interaction nor the three-way interaction was statistically significant, confirming that the effect of lack of control is not different for correct and false identification attempts when the percentage of identification attempts at each level is considered. The nonsignificant three-way interaction also speaks against pure random guessing. If lack of control was increasing random guessing, participants in this group would have produced a significantly larger percentage of erroneous responses for early filtering levels, as compared with the control group.

Finally, if lack of control induced random guessing, participants in this group would have also made more overall false identification attempts. A 2 (identification attempts, correct vs. false) $\times 2$ (group) mixed model ANOVA on the total number of identification attempts showed that only the main effect of identification attempts was statistically significant, $F(1,46)=240.63, p<.0001$, partial $\eta^{2}=.84$, with, overall, more correct than false identification attempts in both groups. Neither the effect of group nor the interaction was significant, confirming that participants in the lack-of-control condition did not make more false identification attempts than did those in the control group.

\section{Discussion}

In the present study, we found that identification of real visual objects is influenced by the need for control, when this motivational force is experimentally induced by creating a sense of lack of control (Whitson \& Galinsky, 2008). Need for control has been found to play a major role in several areas (Cohen, 1986; Pittman \& Pittman, 1979; Roth \& Bootzin, 1974) but has not been assessed previously in the perception of meaningful and familiar visual objects. As compared with control participants, those lacking control made initial identification attempts to visual objects at more degraded versions of the pictures, but this did not decrease accuracy.

The advantage observed in the group in which lack of control was experimentally induced was revealed both by the lower levels of filtering at which first identification attempts occurred (i.e., for figures that were more degraded), as well as by the larger percentage of identification attempts occurring at a lower level of filtering, when LFs were still predominant. Conversely, control participants made more overall identification attempts at filtering levels 4 and 5, when fine HFs started to be predominant.

These findings confirm the results of the Whitson and Galinsky (2008) study, in which the experimental induction of lack of control increased illusory pattern perception in conditions in which pictures had no image and contained only white noise. However, the new findings also add some important new data, since they show that experiencing lack of control not only induces an early visual closure in random visual noise, but also influences the processing of structured meaningful visual objects when they are degraded. This implies that lack of control does not simply make people more prone to impose an illusory structure on random perceptual information, as previously suggested (Whitson \& Galinsky, 2008), but also increases both accurate and inaccurate identification attempts for meaningful visual objects. Thus, our data contribute a new topdown factor that influences identification processesnamely, control. Individuals who lack control may experience visual closure earlier, or they may be more motivated to achieve visual closure, although they do so with no significant change in accuracy.

Earlier responding due to need for control was present for both correct identifications (which were the majority of responses in both groups) and incorrect identification attempts. Correct and incorrect identification attempts share most of the processes involved in object model search and selection. In both cases, perceptual analyses have progressed enough to activate long-term memory representations that share elements with the visual objects. Recent data suggest that both correct and incorrect identification attempts are characterized by the same ERP component (a subcomponent of the N350 complex; Schendan \& Maher, 2009). In the case of correct identifications, the best object model is selected. In the case of incorrect identification attempts, the selected structural model is wrong but shares visual features with the perceptual image. Thus, in our study, incorrect identification attempts referred to objects that were visually similar to the one presented. 
Our findings indicate that experiencing lack of control did not encourage random guessing. If it had, participants in the lack-of-control group would have produced a significantly larger number of erroneous responses for early filtering levels, as compared with the control group, but we did not find this effect. Nor did lack of control induce an overall higher total number of false identification attempts.

These results also do not indicate that need for control leads to an earlier activation of the correct memory representation of the presented object. Were this the case, more correct identification responses would have been obtained in the group needing control than in the control group.

Two different explanations might be advanced for the effect of lack of control on both correct and incorrect identification attempts. First, need for control might move the normal perceptual processes involved in the identification of degraded objects earlier. This might consist in activating more efficient processing, in which less physical information in the visual stimulus is required to trigger adequate (although not necessarily correct) matching with a long-term-memory representation. Conversely, it might consist in an earlier activation of potential candidates in long-term visual memory.

A second explanation needs also to be considered. Lacking control might induce participants to adopt a more liberal response criterion, venturing an identification response even when little information is present. In this case, lack of control would lead not to a more efficient processing of visual stimuli, but to a shift and relaxation in the decisional criterion. In this case, however, one would expect a downward shift in accuracy, which we did not find. Nevertheless, additional future studies are necessary to distinguish between these explanations and to better understand the mechanism underlying the effect of lack of control on behavioral performance. However, our data clearly indicate that, independently of the interpretation, lack of control affects the processes involved in the identification of degraded visual objects.

With respect to previous findings on the modulatory effect of cognitive and motivational top-down processes on visual object identification (e.g., expertise, Viggiano et al., 2006; differences in visual object imagery abilities, Vannucci et al., 2008; needs and expectations. Bruner \& Goodman, 1947; Bruner \& Postman, 1947; Levine et al., 1942), our findings provide evidence for the effect of a new motivational top-down factor (lack of control) on identification processes. In our study, the effect of this top-down variable was assessed in conditions of ambiguity and degradation, when the organizing capacity of the bottomup structural properties intrinsic to the stimuli are reduced, thus favoring the action of top-down processes. Further studies are necessary to investigate whether this effect is also present when visual objects are not degraded, leading, for example, to shorter response times in recognition tasks.

\section{References}

Bruner, J. S., \& Postman, L. (1947). Tension and tension-release as organizing factors in perception. Journal of Personality, 15, 300308.

Bruner, J. S., \& Potter, M. C. (1964). Interference in visual recognition. Science, 144, 424-425.

Cohen, S. (1986). Behavior, health, and environmental stress. New York: Plenum.

Collin, C. A. (2006). Spatial-frequency thresholds for object categorisation at basic and subordinate levels. Perception, 35, 41-52.

Dickerson, J., \& Humphreys, G. W. (1999). On the identification of misoriented objects: Effects of task and level of stimulus description. European Journal of Cognitive Psychology, 11, $145-166$.

Hamm, J. P., \& McMullen, P. A. (1998). Effects of orientation on the identification of rotated objects depend on the level of identity. Journal of Experimental Psychology: Human Perception and Performance, 24, 413-426.

Hughes, H. C., Nozawa, G., \& Kitterle, F. (1996). Global precedence, spatial frequency channels, and the statistics of natural images. Journal of Cognitive Neuroscience, 8, 197-230.

Jolicœur, P. (1985). The time to name disoriented natural objects. Memory \& Cognition, 13, 289-303.

Laws, K. R., \& Gale, T. M. (2002). Category-specific naming and the "visual" characteristics of line drawn stimuli. Cortex, 38, 7-21.

Laws, K. R., Gale, T. M., \& Leeson, V. C. (2003). The influence of surface and edge-based visual similarity on object recognition. Brain and Cognition, 53, 232-234.

Levine, R., Chein, I., \& Murphy, G. (1942). The relation of the intensity of a need to the amount of perceptual distortion. The Journal of Psychology, 13, 283-293.

Lindfield, K. C., \& Wingfield, A. (1999). Recognition of fragmented pictures: Inhibitory connections versus speed of processing. Experimental Aging Research, 25, 223-242.

Lindfield, K. C., Wingfield, A., \& Bowles, N. L. (1994). Identification of fragmented pictures under ascending versus fixed presentation in young and elderly adults: Evidence for the inhibition-deficit hypothesis. Aging and Cognition, 1, 282-291.

Martin, A. (2007). The representation of object concepts in the brain. Annual Review of Psychology, 58, 25-45.

Neuberg, S. L., \& Newsom, J. T. (1993). Personal need for structure: Individual differences in the desire for simple structure. Journal of Personality and Social Psychology, 65, 113-131.

Oliva, A., \& Schyns, P. (2000). Diagnostic colors mediate scene recognition. Cognitive Psychology, 41, 176-210.

Pittman, N. L., \& Pittman, T. S. (1979). Effects of amount of helplessness training and internal-external locus of control on mood and performance. Journal of Personality and Social Psychology, 37, 39-47.

Roth, S., \& Bootzin, R. R. (1974). The effects of experimentally induced expectancies of external control: An investigation of learned helplessness. Journal of Personality and Social Psychology, 29, 253-264.

Schendan, H. E., \& Kutas, M. (2002). Neurophysiological evidence for two processing times for visual object identification. Neuropsychologia, 40, 931-945.

Schendan, H. E., \& Maher, S. M. (2009). Object knowledge during entry-level categorization is activated and modified by implicit memory after 200 ms. Neuroimage, 44, 1423-1438. 
Snodgrass, J. G., \& Hirshman, E. (1991). Theoretical explorations of the Bruner Potter (1964) interference effect. Journal of Memory and Language, 30, 273-293.

Tanaka, J. W., \& Presnell, L. M. (1999). Color diagnosticity in object recognition. Perception \& Psychophysics, 61, 1140-1153.

Tarr, M. J., \& Pinker, S. (1989). Mental rotation and orientation dependence in shape recognition. Cognitive Psychology, 21, 233-283.

Thompson, M. M., Naccarato, M. E., \& Parker, K. E. (1989). Assessing cognitive need: The development of the personal need for structure and personal fear of invalidity scales. Paper presented at the annual meeting of the Canadian Psychological Association, Halifax. Canada: Nova Scotia.

Ullman, S. (1996). High-level vision: Object recognition and visual cognition. Cambridge, MA: MIT Press.

Vannucci, M., Mazzoni, G., Chiorri, C., \& Cioli, L. (2008). Object imagery and object identification: Object imagers are better at identifying spatially-filtered visual objects. Cognitive Processing, 9, 137-143.

Vannucci, M., \& Viggiano, M. P. (2000). Category effects on the processing of plane-rotated objects. Perception, 29, 287302.

Vannucci, M., Viggiano, M. P., \& Argenti, F. (2001). Identification of spatially filtered stimuli as function of semantic categories. Cognitive Brain Research, 12, 475-478.

Viggiano, M. P., Righi, S., \& Galli, G. (2006). Category-specific visual recognition as affected by aging and expertise. Archives of Gerontology and Geriatrics, 42, 329-338.

Viggiano, M. P., Vannucci, M., \& Righi, S. (2004). A new standardised set of ecological pictures for experimental and clinical research on visual object processing. Cortex, 40, 491-509.

Whitson, J. A., \& Galinsky, A. D. (2008). Lacking control increases illusory pattern perception. Science, 322, 115-117. 